\section{Tracking Control of Limit Cycle Oscillations in an Aero-Elastic System}

\author{
B. J. Bialy ${ }^{1}$ \\ Department of Mechanical and Aerospace Engineering, \\ University of Florida, \\ Gainesville, FL 32611 \\ e-mail: bialybj@ufl.edu
}

\section{Crystal L. Pasiliao}

Air Force Research Laboratory,

Munitions Directorate,

Eglin AFB, FL 32542

e-mail: crystal.pasiliao@eglin.af.mil

\author{
H. T. Dinh \\ Department of Mechanical and Aerospace Engineering, \\ University of Florida, \\ Gainesville, FL 32611 \\ e-mail: huyentdinh@ufl.edu
}

\author{
W. E. Dixon \\ Department of Mechanical and Aerospace Engineering, \\ University of Florida, \\ Gainesville, FL 32611 \\ e-mail: wdixon@ufl.edu
}

Limit cycle oscillations (LCOs) affect current fighter aircraft and are expected to be present on next generation fighter aircraft. Current efforts in control systems designed to suppress LCO behavior have either used a linear model, restricting the flight regime, require exact knowledge of the system dynamics, or require uncertainties in the system dynamics to be linear-in-theparameters and only present in the torsional stiffness. Furthermore, the aerodynamic model used in prior research efforts neglects nonlinear effects. This paper presents the development of a controller consisting of a continuous robust integral of the sign of the error (RISE) feedback term with a neural network (NN) feedforward term to achieve asymptotic tracking of uncertainties that do not satisfy the linear-in-the-parameters assumption. Simulation results are presented to validate the performance of the developed controller. [DOI: 10.1115/1.4027946]

\section{Introduction}

LCOs commonly occur and remain as a problem on high performance fighter aircraft [1]. Typically, LCO behavior consists of antisymmetric motion of the wing and lateral motion of the fuselage. It may be self-induced or initiated through control inputs; however, once started, the motion is self-sustaining and persists until the flight conditions have been sufficiently altered. LCO behavior is considered to be closely related to flutter, except that coupling between the nonlinearities in the structural response and the unsteady aerodynamic forces yields a limited amplitude motion [2]. The major concern with LCO is the impact on the pilot's ability to successfully complete the mission in a safe and effective manner. Specifically, the lateral motion of the fuselage

${ }^{1}$ Corresponding author.

Contributed by the Dynamic Systems Division of ASME for publication in the Journal of Dynamic Systems, Measurement, and Control. Manuscript received July 18, 2013; final manuscript received June 25, 2014; published online August 8, 2014. Assoc. Editor: Yang Shi. experiencing LCO may cause the pilot to have difficulty reading cockpit instruments and can lead to the termination of the mission or the avoidance of a part of the flight envelope critical to combat survivability. Additionally, the effects of LCO on ordnance are questionable [2]. These questions include the ability of the ordnance to be safely released during LCO, the effects on target acquisition for smart munitions, and the effects on the accuracy of unguided weapons.

Several control strategies have been developed to suppress LCO behavior in aero-elastic systems requiring knowledge of the dynamics [3-10]. In recent years, multiple adaptive controllers have been developed to compensate for uncertainties in the torsional stiffness model. An adaptive control strategy was designed in Ref. [11] for a structurally nonlinear wing section with a single trailing edge control surface. The design assumes linearin-the-parameters structural nonlinearities and uses feedback linearization to compensate for a portion of the system dynamics. Experimental results on a two degree of freedom wing section using the adaptive controller developed in Ref. [11] and the linear controller developed in Ref. [3] were presented in Ref. [12]. The results indicate that the adaptive controller was capable of suppressing the LCO behavior at velocities up to $23 \%$ higher than the flutter velocity. A model reference adaptive control strategy was developed in Ref. [13] to suppress LCO behavior of a typical wing section with linear-in-the-parameters uncertainties in the pitch stiffness model. A feedback linearization control strategy that uses multiple control surfaces was developed in Ref. [14] to mitigate LCO behavior on a typical wing section with the same uncertainties as in Ref. [11]. The controller showed improved transient performance and was capable of stabilizing the wing section at higher freestream velocities when compared with Ref. [13].

Previously developed controllers either use linearized system dynamics and are restricted to specific flight regimes, require exact knowledge of the system dynamics, or consider only uncertainties in the dynamics that satisfy the linear-in-the-parameters assumption. When any of these conditions are not met, the previously developed controllers can no longer guarantee stability. The objective in this paper is to develop a controller (based on the preliminary work by the authors [15]) to suppress LCO behavior in an uncertain two degree of freedom airfoil section with an attached store, one control surface, and an additive unknown nonlinear disturbance that does not satisfy the linear-in-theparameters assumption. The unknown disturbance represents unsteady nonlinear aerodynamic effects. A NN is used as a feedforward control term to compensate for the unknown nonlinear disturbance and a RISE feedback term [16-18] ensures asymptotic tracking of a desired state trajectory. A RISE-like controller was also recently developed in Ref. [19] where the controller is embedded in hyperbolic saturation functions to ensure that the actuator constraints are not breached.

\section{Aero-Elastic System Model}

The subsequent development and stability analysis are based on an aero-elastic model given by [20]

$$
F(q, \dot{q}, \delta)=M(q) \ddot{q}+C(q, \dot{q}) \dot{q}+K(q) q-N
$$

where $q \triangleq\left[\begin{array}{ll}h & \alpha\end{array}\right]^{\mathrm{T}} \in \mathbb{R}^{2}$ is a composite vector of the vertical position and angle of attack (AOA) of the wing-store section, respectively.

Based on the description of LCO behavior, the system states can be bounded as $\|q\| \leq \kappa_{1},\|\dot{q}\| \leq \kappa_{2}$, and $\|\ddot{q}\| \leq \kappa_{3}$, where $\kappa_{1}, \kappa_{2}, \kappa_{3} \in \mathbb{R}$ are known positive constants. In Eq. (1), $M \in \mathbb{R}^{2 \times 2}, C \in \mathbb{R}^{2 \times 2}, K \in \mathbb{R}^{2 \times 2}, F \in \mathbb{R}^{2}$, and $N \in \mathbb{R}^{2}$ are defined as

$$
\begin{aligned}
& M \triangleq\left[\begin{array}{ll}
m_{1} & m_{2} \\
m_{2} & m_{4}
\end{array}\right], \quad C \triangleq\left[\begin{array}{cc}
c_{h_{1}} & c_{h_{2}} \dot{\alpha} \\
0 & c_{\alpha}
\end{array}\right], \quad K \triangleq\left[\begin{array}{cc}
k_{h} & 0 \\
0 & k_{\alpha}
\end{array}\right], \\
& F \triangleq\left[\begin{array}{l}
-L \\
P_{M}
\end{array}\right], \quad N \triangleq\left[\begin{array}{l}
N_{1} \\
N_{2}
\end{array}\right]
\end{aligned}
$$


The elements of the mass matrix in Eq. (2) are the unknown constants $m_{1}, m_{4} \in \mathbb{R}$, and $m_{2} \in \mathbb{R}$, which are explicitly defined in Ref. [15]. In Eq. (2), $c_{h_{1}}, c_{\alpha} \in \mathbb{R}$ are the unknown constant damping coefficients of the plunge and pitch motion, respectively, and $\quad c_{h_{2}} \in \mathbb{R} \quad$ is defined as $c_{h_{2}}(q) \triangleq-\left(r_{x}-a\right) m_{w} b \cos (\alpha)$ $-\left(r_{h}-a_{h}\right) m_{w} b \sin (\alpha)-\left(s_{x}-a\right) m_{s} b \cos (\alpha)-\left(s_{h}-a_{h}\right) m_{s} b \sin (\alpha)$. The unknown constant plunge stiffness coefficient in Eq. (2) is denoted by $k_{h} \in \mathbb{R}$, and the unknown nonlinear pitch stiffness coefficient in Eq. (2) is denoted by $k_{\alpha}(q) \in \mathbb{R}$ and is modeled as a fourth-order polynomial with uncertain constant coefficients. Also in Eq. (2), $L \in \mathbb{R}$ and $P_{M} \in \mathbb{R}$ are the lift force and pitch moment acting on the wing-store section, respectively, and are modeled as

$$
\begin{gathered}
L=\rho U^{2} b S C_{l_{\alpha}} \alpha_{\mathrm{ef}}+C_{l_{\delta}} \delta \\
P_{M}=\rho U^{2} b^{2} S C_{l_{\alpha}}\left(\frac{1}{2}+a\right) \alpha_{\mathrm{ef}}+C_{m_{\delta}} \delta
\end{gathered}
$$

where $\rho, U, S, C_{l_{\alpha}}, C_{l_{\delta}}$, and $C_{m_{\delta}} \in \mathbb{R}$ are unknown constant coefficients. In Eqs. (3) and (4), $\delta(t) \in \mathbb{R}$ is the control surface deflection angle, and $\alpha_{\mathrm{ef}} \in \mathbb{R}$ is defined as $\alpha_{\mathrm{ef}} \triangleq \alpha+(\dot{h} / U)$ $+(b((1 / 2)-a) \dot{\alpha} / U)$. Moreover, the terms in Eq. (2), $N_{1}, N_{2} \in \mathbb{R}$ are unknown nonlinear disturbances that represent unmodeled nonlinear aerodynamics such as shock effects.

\section{Control Objective}

The control objective is to guarantee the wing section AOA, $\alpha$, tracks a desired trajectory defined as $\alpha_{d} \in \mathbb{R}$. The formulation of an AOA tracking problem enables the AOA of the wing to be optimized for a given metric and flight condition. The control development and analysis are based on the assumption that $\alpha_{\mathrm{d}}, \dot{\alpha}_{\mathrm{d}}, \ddot{\alpha}_{\mathrm{d}}, \dddot{\alpha}_{\mathrm{d}}, \dddot{\alpha}_{\mathrm{d}}, \in \mathscr{L}_{\infty}$. To quantify the control objective and facilitate the control design, a tracking error, $e_{1} \in \mathbb{R}$, and two auxiliary tracking errors, $e_{2}, r \in \mathbb{R}$, are defined as

$$
\begin{gathered}
e_{1} \stackrel{\Delta}{=} \alpha-\alpha_{\mathrm{d}} \\
e_{2} \triangleq \dot{e}_{1}+\gamma_{1} e_{1} \\
r \stackrel{\Delta}{=} \dot{e}_{2}+\gamma_{2} e_{2}
\end{gathered}
$$

where $\gamma_{1}, \gamma_{2} \in \mathbb{R}$ are positive constants. The subsequent development is based on the assumption that $q$ and $\dot{q}$ are measurable. Hence, the auxiliary tracking error, $r$, is not measurable, since it depends on $\ddot{q}$. Substituting the system dynamics in Eq. (1) into the error dynamics in Eq. (7) yields the following expression: $r=f+g \delta$, where the auxiliary terms $f \in \mathbb{R}$ and $g \in \mathbb{R}$ are defined as

$$
\begin{gathered}
f=-\frac{m_{2}}{\operatorname{det}(M)}\left(-\tilde{C}_{11} \dot{h}-\tilde{C}_{12} \dot{\alpha}-\tilde{K}_{11} h-\tilde{K}_{12} \alpha+N_{1}\right) \\
+\frac{m_{1}}{\operatorname{det}(M)}\left(-\tilde{C}_{21} \dot{h}-\tilde{C}_{22} \dot{\alpha}-\tilde{K}_{22} \alpha+N_{2}\right) \\
-\ddot{\alpha}_{\mathrm{d}}+\gamma_{1} \dot{e}_{1}+\gamma_{2} e_{2} \\
g=\frac{m_{2}}{\operatorname{det}(M)} C_{l_{\delta}}+\frac{m_{1}}{\operatorname{det}(M)} C_{m_{\delta}}
\end{gathered}
$$

and $g$ is a positive term provided that sufficient conditions on the wing geometry and store location are met. $^{2}$

${ }^{2}$ See [15] for details.

\section{Control Development}

After some algebraic manipulation, the open-loop error system for $r$ can be obtained as $(1 / g) r=\chi+\left(f_{\mathrm{d}} / g_{\mathrm{d}}\right)+\delta$, where $g_{\mathrm{d}} \in \mathbb{R}$ and $f_{\mathrm{d}} \in \mathbb{R}$ are defined as

$$
\begin{gathered}
f_{\mathrm{d}}=-\frac{m_{2}\left(q_{\mathrm{d}}\right)}{\operatorname{det}\left(M\left(q_{\mathrm{d}}\right)\right)}\left(-\tilde{C}_{11} \dot{h}_{\mathrm{d}}-\tilde{C}_{12}\left(q_{\mathrm{d}}, \dot{q}_{\mathrm{d}}\right) \dot{\alpha}_{\mathrm{d}}-\tilde{K}_{11} h_{\mathrm{d}}-\tilde{K}_{12} \alpha_{\mathrm{d}}\right) \\
+\frac{m_{1}}{\operatorname{det}\left(M\left(q_{\mathrm{d}}\right)\right)}\left(-\tilde{C}_{21} \dot{h}_{\mathrm{d}}-\tilde{C}_{22} \dot{\alpha}_{\mathrm{d}}-\tilde{K}_{22}\left(q_{\mathrm{d}}\right) \alpha_{\mathrm{d}}\right)-\ddot{\alpha}_{\mathrm{d}} \\
g_{\mathrm{d}}=\frac{m_{2}\left(q_{\mathrm{d}}\right)}{\operatorname{det}\left(M\left(q_{\mathrm{d}}\right)\right)} C_{l_{\delta}}+\frac{m_{1}}{\operatorname{det}\left(M\left(q_{\mathrm{d}}\right)\right)} C_{m_{\delta}}
\end{gathered}
$$

where $q_{\mathrm{d}} \triangleq\left[\begin{array}{ll}h_{\mathrm{d}} & \alpha_{\mathrm{d}}\end{array}\right]^{\mathrm{T}} \in \mathbb{R}^{2}$ and $h_{\mathrm{d}} \in \mathbb{R}$ is a desired trajectory for the vertical position of the wing. The subsequent development is based on the assumption that the designed trajectory, $h_{\mathrm{d}}$ and $\dot{h}_{\mathrm{d}}$, are bounded. Based on the open-loop error system and the stability analysis in Ref. [15], the control surface deflection angle is designed as $\delta=-\left(\widehat{f_{\mathrm{d}}} / g_{\mathrm{d}}\right)-\mu$, where $\left(\widehat{f_{\mathrm{d}}} / g_{\mathrm{d}}\right) \in \mathbb{R}$ is defined as $\left(\widehat{f}_{\mathrm{d}} / g_{\mathrm{d}}\right) \triangleq \hat{W}^{\mathrm{T}} \sigma\left(\hat{V}^{\mathrm{T}} x_{\mathrm{d}}\right)$, and $\mu \in \mathbb{R}$ denotes the subsequently defined RISE feedback term. In the control design, $\hat{W} \in \mathbb{R}^{n_{2}+1}$ and $\hat{V} \in \mathbb{R}^{7 \times n_{2}}$ denote estimates for the ideal weight matrices whose update laws are defined as $\dot{\hat{W}} \triangleq \operatorname{proj}\left(\Gamma_{1} \hat{\sigma}^{\prime} \hat{V}^{\mathrm{T}} \dot{x}_{d} e_{2}\right)$ and $\dot{\hat{V}} \triangleq \operatorname{proj}\left(\Gamma_{2} \dot{x}_{\mathrm{d}}\left(\hat{\sigma}^{\prime \mathrm{T}} \hat{W} e_{2}\right)^{\mathrm{T}}\right), \quad$ where $\quad \Gamma_{1} \in \mathbb{R}^{\left(n_{2}+1\right) \times\left(n_{2}+1\right)}$ and $\Gamma_{2}$ $\in \mathbb{R}^{7 \times 7}$ are constant and positive definite control matrices, and $\operatorname{proj}(\cdot)$ denotes a smooth projection algorithm in Ref. [21]. The RISE feedback term is defined as $\mu \triangleq\left(k_{s_{1}}+k_{s_{2}}\right) e_{2}-\left(k_{s_{1}}+k_{s_{2}}\right)$ $e_{2}(0)+\nu$, where $\nu \in \mathbb{R}$ is the Filippov solution to the following differential equation $\dot{\nu}=\left(k_{s_{1}}+k_{s_{2}}\right) \gamma_{2} e_{2}+\beta_{1} \operatorname{sgn}\left(e_{2}\right)$ and $k_{s_{1}}$, $k_{s_{2}}, \beta_{1} \in \mathbb{R}$ are positive constant control gains.

\section{Stability Analysis}

See Ref. [15] for details.

\section{Simulation Results}

A numerical simulation is presented to illustrate the performance of the developed controller and provide a comparison with the controller in Ref. [12]. The controller from Ref. [12] was selected for comparison because it is one of the few controllers that consider structural uncertainties. However, this is not an equal comparison, since the controller in Ref. [12] considers uncertainties in the pitch stiffness only, while the control strategy developed in this paper considers uncertainties in all parameters in the structural and aerodynamic models. For this reason, the structural and aerodynamic parameters that are assumed to be known in Ref. [12] are taken to be off by $10 \%$ from the actual values. The controller in Ref. [12] is given by $\delta=\left(1 / g_{4} U^{2}\right)\left(-F(q, \dot{q})-\hat{\Theta}^{\mathrm{T}}\right.$ $\left.R(q)-\bar{k}_{1} \alpha-\bar{k}_{2} \dot{\alpha}\right)$, where $g_{4} \in \mathbb{R}$ is a control effectiveness parameter, $U \in \mathbb{R}$ denotes the freestream velocity, $F(q, \dot{q}) \in \mathbb{R}$ is a feedback linearization term that requires exact model knowledge of certain parameters in the structural model and all parameters in the aerodynamic model, $\hat{\Theta} \in \mathbb{R}^{i}$ denotes a vector of the estimates of the uncertain parameters in the pitch stiffness model, $R(q) \in \mathbb{R}^{i}$ represents a known regression matrix, and $\bar{k}_{1}, \overline{k_{2}} \in \mathbb{R}$ are positive control gains. The control gains were selected as $\bar{k}_{1}=\bar{k}_{2}=60$ based on improving the transient performance of the controller while keeping the control effort within tolerable limits ( $\pm 10 \mathrm{deg}$ ). The estimate, $\hat{\Theta}$, is updated via a gradient update law given by $\dot{\hat{\Theta}}=\dot{\alpha} R^{\mathrm{T}}(q)$. The model parameters for the simulation can be found in Ref. [15]. The control objective is to regulate the AOA to $0 \mathrm{deg}$ from the initial condition $h(0)=0 \mathrm{~m}$, $\dot{h}(0)=0 \mathrm{~m} / \mathrm{s}, \alpha(0)=3.0 \mathrm{deg}$, and $\dot{\alpha}(0)=0 \mathrm{deg} / \mathrm{s}$. From Fig. 1 , it is evident that the system, under the above conditions, experiences LCO behavior in the absence of a control strategy and 

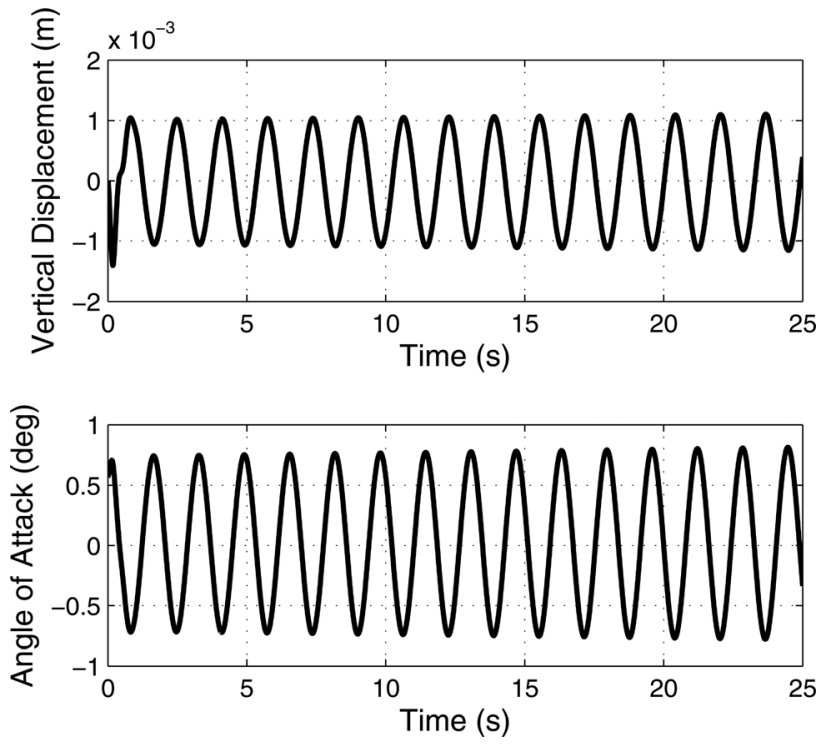

Fig. 1 Aero-elastic system free response without disturbances
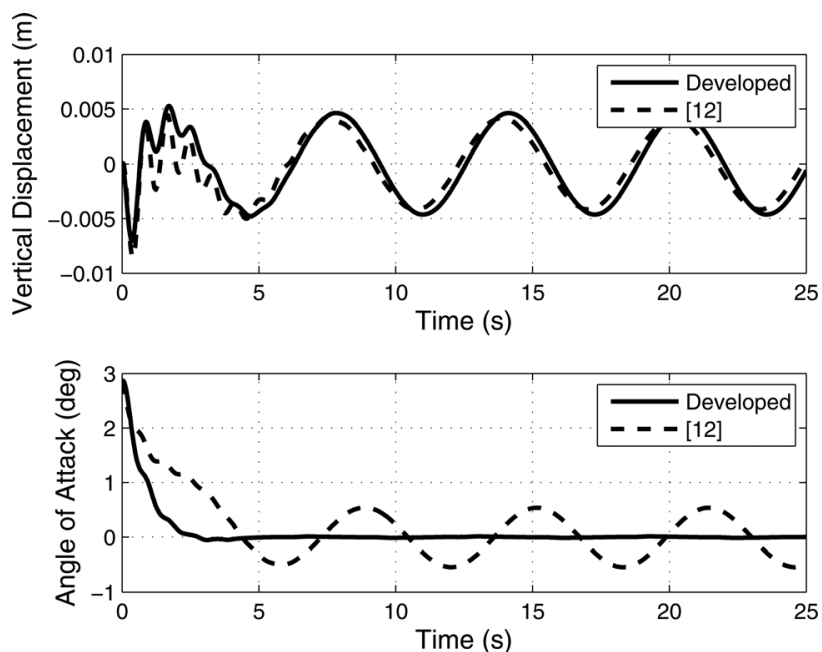

Fig. 2 Aero-elastic system states in the presence of an additive disturbance

exogenous disturbances. The developed control strategy was applied to the system in the absence of exogenous disturbances with the following gains: $\gamma_{1}=2, \gamma_{2}=3, k_{s_{1}}+k_{s_{2}}=3, \beta_{1}=0.1$, $n_{2}=25, \Gamma_{1}=10 I_{26}$, and $\Gamma_{2}=10 I_{7}$, where $I_{m}$ denotes an $m \times m$ identity matrix.

The two controllers were applied to the system in the presence of an additive exogenous disturbance selected as

$$
N(t)=\left[\begin{array}{ll}
0.25 \cos (t) & 0.25 \sin (t)
\end{array}\right]^{\mathrm{T}}
$$

Figures 2 and 3 show the system states and control effort in the presence of the additive disturbance, respectively. The developed controller is capable of regulating the AOA of the wing section in the presence of exogenous disturbances with control surface deflections that remain within tolerable limits. However, the controller in Ref. [12] is not capable of eliminating the effects of the disturbance in the wing section vertical position. Due to the coupled nature of the aero-elastic system dynamics and the availability of a single control surface, any disturbance in the AOA will propagate into the vertical position as an unmatched disturbance. One solution to this issue is to include an additional control

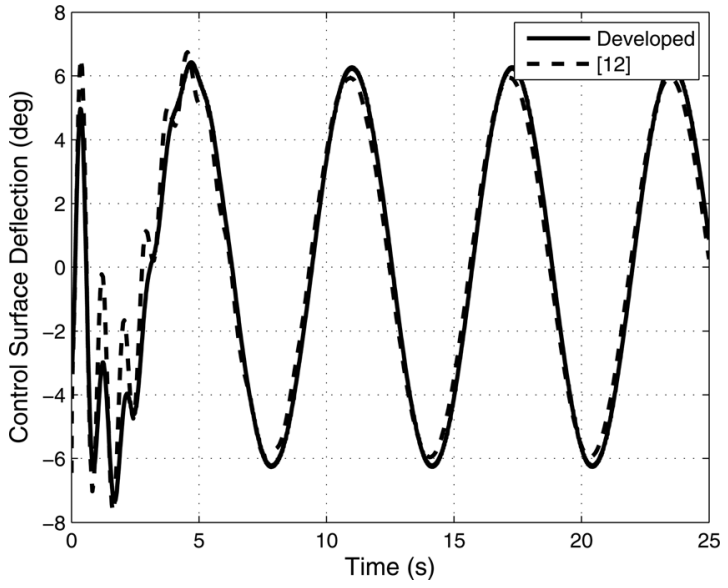

Fig. 3 Control surface deflection, $\delta(t)$, for the developed controller and the controller from Ref. [12]

Table 1 Monte Carlo simulation results

\begin{tabular}{lll}
\hline \hline & Mean & Standard deviation \\
\hline Maximum error & $2.9 \mathrm{deg}$ & $0.0038 \mathrm{deg}$ \\
RMS error & $0.97 \mathrm{deg}$ & $0.073 \mathrm{deg}$ \\
Maximum control effort & $7.5 \mathrm{deg}$ & $2.6 \mathrm{deg}$ \\
Settling time & $3.4 \mathrm{~s}$ & $0.39 \mathrm{~s}$ \\
\hline
\end{tabular}

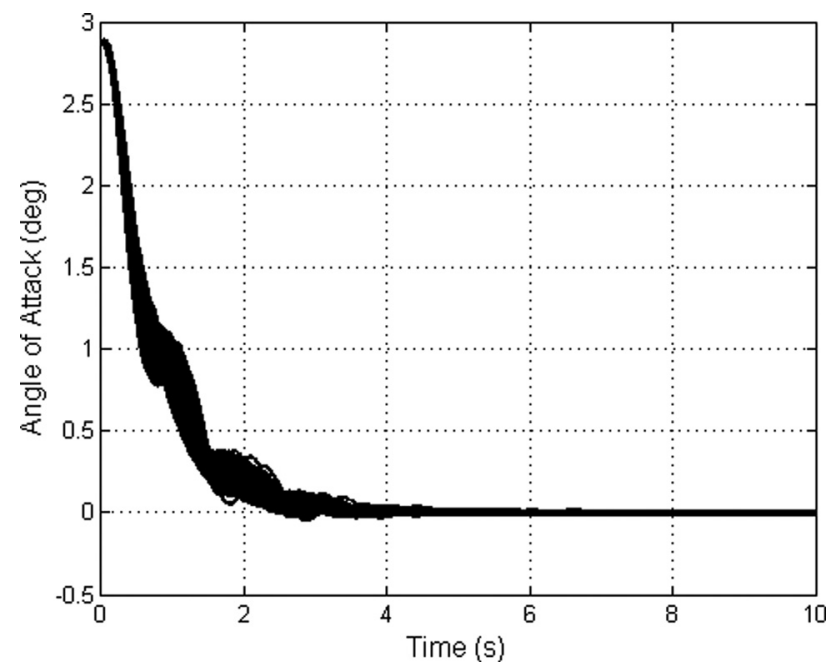

Fig. 4 Monte Carlo AOA trajectories

surface at the leading edge that could be used to suppress unwanted motion in the vertical position.

A 1500 sample Monte Carlo simulation was executed to demonstrate the robustness of the developed controller to plant uncertainties and sensor noise. The uncertain model parameters were uniformly distributed over a range that extended from $80 \%$ to $120 \%$ of the nominal values found in Ref. [15]. A zero mean noise signal uniformly distributed over an interval was added to each measurement. For the vertical displacement and velocity, the interval was $\pm 2.5 \times 10^{-6} \mathrm{~m}$ and $\pm 2.5 \times 10^{-4} \mathrm{~m} / \mathrm{s}$, respectively. For the AOA and AOA rate, the interval was $\pm 4.5 \times 10^{-3} \mathrm{rad}$ and $\pm 1 \times 10^{-3} \mathrm{rad} / \mathrm{s}$. For each sample, the maximum of the absolute value of the tracking error and control surface deflection, the RMS value of the tracking error, and the settling time were calculated. The settling time was defined to be the time at which the 


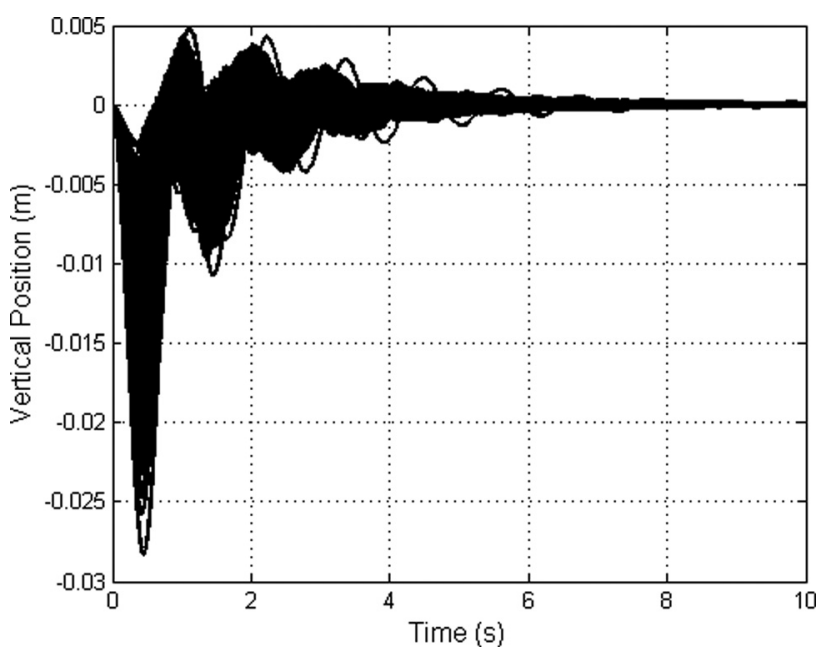

Fig. 5 Monte Carlo vertical position trajectories

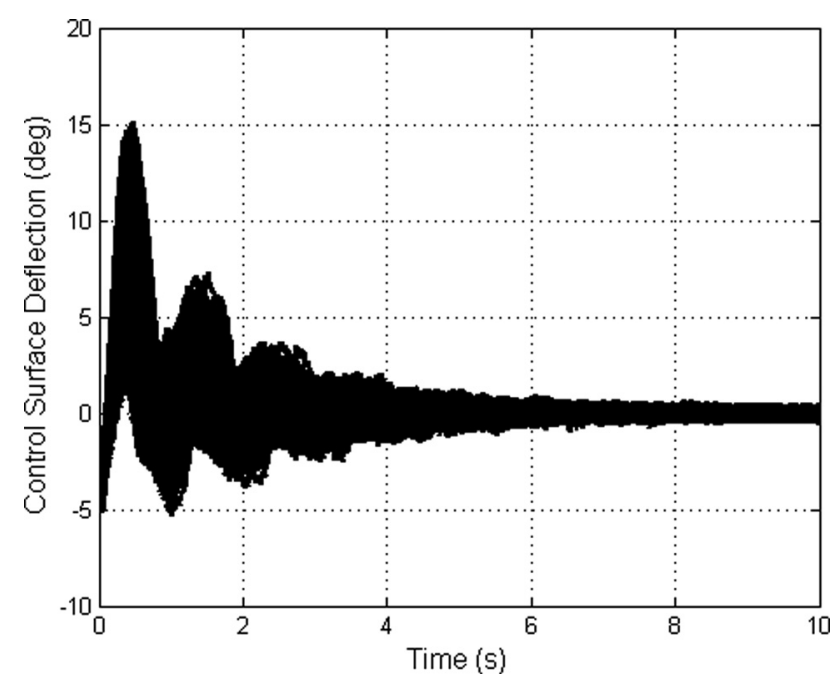

Fig. 6 Monte Carlo control effort

tracking error remained within $\pm 0.01 \mathrm{deg}$. The results, presented in Table 1, indicate that the maximum error and RMS error of the system do not vary significantly over the range of the uncertainties considered.

Figures 4-6 show the system states and control effort for all 1500 Monte Carlo samples. Figure 4 shows that the AOA for all samples converges to zero in approximately $3.5 \mathrm{~s}$ and the small scatter of the individual trajectories indicates that the system performance is not significantly impacted by variations in the uncertain parameters. It is evident from Fig. 5 that the uncontrolled vertical displacement damps out for all samples. Figure 6 shows that the control surface deflection for all samples remains within tolerable limits. However, the maximum control effort over all Monte Carlo simulations is approximately twice that of the numerical result shown in Fig. 3. This sensitivity indicates that in a more severe LCO, variations in the uncertain parameters could lead to a control effort greater than the actuator limits.

\section{Conclusion}

A robust adaptive control strategy is developed to suppress LCO behavior of an aero-elastic system. The developed controller uses a NN feedforward term to account for structural and aerodynamic uncertainties, and a RISE feedback term to guarantee asymptotic tracking of a desired AOA trajectory. A Lyapunov- based stability analysis is used to prove asymptotic tracking. Simulations illustrate LCO suppression and AOA tracking performance over a range of uncertainty. A potential drawback to the developed control strategy is that the control law does not account for actuator limits. As the severity of the LCO behavior increases, the developed controller can demand a large control surface deflection. Additionally, the Monte Carlo simulation results indicated that the maximum control effort is sensitive to variations in the parameter uncertainties, which could lead to unexpected actuator saturation. Efforts in Ref. [19] could be applied to incorporate saturation limits.

Currently, there is a lack of clarity amongst researchers concerning the driving mechanism of LCO behavior. The current work, as in Ref. [20], capture LCO symptoms through the introduction of a nonlinear torsional stiffness. Future efforts are focused on extending the developed controller to a flexible wing modeled as a bending and twisting cantilever beam. As knowledge of the driving mechanism is furthered, it could be included into the flexible wing model and developed boundary control techniques to provide a more complete control strategy for LCO suppression.

\section{Acknowledgment}

This research is supported by a contract with the Air Force Research Laboratory, Munitions Directorate at Eglin AFB and the Air Force Office of Scientific Research Test and Evaluation portfolio. Any opinions, findings, and conclusions or recommendations expressed in this material are those of the author(s) and do not necessarily reflect the views of the sponsoring agency.

\section{References}

[1] Beran, P. S., Strganac, T. W., Kim, K., and Nichkawde, C., 2004, "Studies of Store-Induced Limit Cycle Oscillations Using a Model With Full System Nonlinearities," Nonlinear Dyn., 37(4), pp. 323-339.

[2] Bunton, R. W., and Denegri, Jr., C. M., 2000, "Limit Cycle Oscillation Characteristics of Fighter Aircraft," J. Aircraft, 37(5), pp. 916-918.

[3] Block, J. J., and Strganac, T. W., 1999, "Applied Active Control for a Nonlinear Aeroelastic Structure,” AIAA J. Guid. Contr. Dynam., 21(6), pp. 838-845.

[4] Ko, J., Strganac, T. W., and Kurdila, A., 1998, "Stability and Control of a Structurally Nonlinear Aeroelastic System,” AIAA J. Guid. Contr. Dynam., 21(5), pp. 718-725.

[5] Zhang, W., and Ye, Z., 2007, "Control Law Design for Transonic Aeroservoelasticity," Aerospace Sci. Technol., 11(2-3), pp. 136-145.

[6] Danowsky, B. P., Thompson, P. M., Farhat, C., Lieu, T., Harris, C., and Lechniak, J., 2010, "Incorporation of Feedback Control Into a HighFidelity Aeroservoelastic Fighter Aircraft Model," J. Aircraft, 47(4), pp. 1274-1282.

[7] Thompson, P. M., Danowsky, B. P., Farhat, C., Lieu, T., Lechniak, J., and Harris, C., 2011, "High-Fidelity Aeroservoelastic Predictive Analysis Capability Incorporating Rigid Body Dynamics," AIAA Paper No. 2011-6209.

[8] Cavagna, L., Ricci, S., and Scotti, A., 2009, "Active Aeroelastic Control Over a Four Control Surface Wing Model,” Aerospace Sci. Technol., 13(7), pp. 374-382.

[9] Prime, Z., Cazzolato, B., Doolan, C., and Strganac, T., 2010, "Linear-Parameter-Varying Control of an Improved Three-Degree-of-Freedom Aeroelastic Model," AIAA J. Guid. Contr. Dynam., 33(2), pp. 615-618.

[10] Elhami, M. R., and Narab, M. F., 2012, "Comparison of SDRE and SMC Control Approaches for Flutter Suppression in a Nonlinear Wing Section," Proceedings of American Control Conference, Montreal, QC, Canada, pp. 6148-6153.

[11] Ko, J., Strganac, T. W., and Kurdila, A., 1999, "Adaptive Feedback Linearization for the Control of a Typical Wing Section With Structural Nonlinearity," Nonlinear Dyn., 18(3), pp. 289-301.

[12] Strganac, T. W., Ko, J., Thompson, D. E., and Kurdila, A., 2000, "Identification and Control of Limit Cycle Oscillations in Aeroelastic Systems," AIAA J. Guid. Contr. Dynam., 23(6), pp. 1127-1133.

[13] Ko, J., Strganac, T. W., Junkins, J. L., Akella, M. R., and Kurdila, A., 2002, "Structured Model Reference Adaptive Control for a Wing Section With Structural Nonlinearity," J. Vib. Control, 8(5), pp. 553-573.

[14] Platanitis, G., and Strganac, T. W., 2004, "Control of a Nonlinear Wing Section Using Leading- and Trailing-Edge Surfaces," AIAA J. Guid. Contr. Dynam., 27(1), pp. 52-58.

[15] Bialy, B. J., Pasiliao, C. L., Dinh, H. T., and Dixon, W. E., 2012, "LyapunovBased Tracking of Store-Induced Limit Cycle Oscillations in an Aeroelastic System," ASME Paper No. DSCC2012-MOVIC2012-8662.

[16] Patre, P. M., MacKunis, W., Kaiser, K., and Dixon, W. E., 2008, "Asymptotic Tracking for Uncertain Dynamic Systems Via a Multilayer Neural Network 
Feedforward and RISE Feedback Control Structure," IEEE Trans. Automat. Control, 53(9), pp. 2180-2185.

[17] Patre, P., Mackunis, W., Dupree, K., and Dixon, W. E., 2011, "Modular Adaptive Control of Uncertain Euler-Lagrange Systems With Additive Disturbances," IEEE Trans. Automat. Control, 56(1), pp. 155-160.

[18] MacKunis, W., Patre, P., Kaiser, M., and Dixon, W. E., 2010, "Asymptotic Tracking for Aircraft Via Robust and Adaptive Dynamic Inversion Methods," IEEE Trans. Control Syst. Technol., 18(6), pp. 1448-1456.
[19] Bialy, B., Andrews, L., Curtis, J. W., and Dixon, W. E., 2014, "Saturated Tracking Control of Store-Induced Limit Cycle Oscillations," AIAA J. Guid. Control Dyn. 37(4), pp. 1316-1323.

[20] Thompson, Jr., D. E., and Strganac, T. W., 2005, "Nonlinear Analysis of Store-Induced Limit Cycle Oscillations," Nonlinear Dyn., 39(1-2), pp. $159-178$.

[21] Krstic, M., Kokotovic, P. V., and Kanellakopoulos, I., 1995, Nonlinear and Adaptive Control Design, John Wiley \& Sons, New York. 